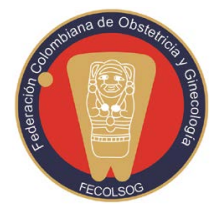

\title{
PERSPECTIVA DE LAS PARTERAS EN JALISCO, MÉXICO, FRENTE AL EMBARAZO DE ALTO RIESGO: ESTUDIO CUALITATIVO
}

\section{Perspectives of midwives in Jalisco, México, regarding high risk pregnancy: A qualiitative study}

Jorge Laureano-Eugenio, MSc ${ }^{1}$; Martha Leticia Mejía-Mendoza, MD, MSc'; Roberto Carlos Ortiz-Villalobos, $\mathrm{MD}^{3}$; Jaime Alejandro Saavedra-Serrano, $\mathrm{MD}^{4}$

Recibido: abril 19/16 - Aceptado: marzo 10/17

\section{RESUMEN}

Objetivo: comprender la perspectiva de las parteras frente a los factores de riesgo, la detección y el manejo del embarazo de alto riesgo en Jalisco, México, 2013.

Materiales y métodos: estudio cualitativo fenomenológico desarrollado durante 2013; a partir del Censo Estatal de Parteras se aplicó muestreo teórico, definiendo tamaño de muestra por saturación teórica del eje: factores de riesgo, detección y manejo del embarazo de alto riesgo. El trabajo de campo inició con la identificación de informantes clave, quienes invitaron a parteras a un "Encuentro

1 Maestro en Ciencias de la Salud Pública; investigador, asesor y promotor de metodología cualitativa en la Secretaría de Salud Jalisco, Guadalajara, Jalisco (México). georgelaure_1@hotmail.com

2 Maestra en Ciencias de la Salud Pública. Docente del Centro Universitario Ciencias de la Salud, Universidad de Guadalajara; médica investigadora de la Secretaría de Salud Jalisco, Guadalajara, Jalisco (México).itzia060500@hotmail.com

3 Especialista en ginecología y obstetricia. Coordinador del área de obstetricia, Hospital General de Occidente, Secretaría de Salud Jalisco; docente del Centro Universitario Ciencias de la Salud, Universidad de Guadalajara Guadalajara, Jalisco (México).robertgine08@hotmail.com

4 Especialista en ginecología y obstetricia. Docente del Centro Universitario Ciencias de la Salud, Universidad de Guadalajara; coordinador médico área normativa "B", Secretaría de Salud Jalisco, Guadalajara, Jalisco (México). saalex_0227@hotmail.com estatal"; se trabajó con las técnicas lluvia de ideas y entrevista grupal; posteriormente se llevaron a cabo 11 entrevistas a profundidad a parteras, realizando análisis semiótico de los datos.

Resultados: fueron 82 parteras informantes, mediana de edad 53 años, parteras tradicionales empíricas $21,95 \%$, parteras tradicionales capacitadas $42,69 \%$ y parteras enfermeras 35,36\%; 59,75\% tienen 20 años o más como parteras, 63,42\% con estudios de primaria o menos y 78,05\% con más de 10 años sin recibir capacitación institucional. Cualitativamente, el embarazo de alto riesgo para parteras enfermeras implica complicaciones obstétricas o neonatales y el control prenatal debe ser en hospitales. En parteras tradicionales, empíricas y capacitadas, su perspectiva tiene elementos de la medicina institucional y tradicional, por lo que señalan que envían al médico a estas mujeres, asumiendo no poder atender estos embarazos; en parteras tradicionales de población indígena representa un embarazo "de peligro", configurado en el modelo mágico-religioso de la salud.

Conclusiones: la perspectiva de las parteras frente al embarazo de alto riesgo está determinada por la medicina tradicional y algunos elementos de la 
medicina institucional, diferenciada según tipo de partera y zona geográfica de desempeño, señalando la disposición de formarse para alcanzar embarazos saludables y sin riesgos.

Palabras clave: partería, embarazo de alto riesgo, salud materna, análisis cualitativo.

\section{ABSTRACT}

Objective: To understand midwife perspective regarding risk factors, detection and management of high risk pregnancy in Jalisco, Mexico, 2013.

Materials and methods: Qualitative phenomenological study conducted during the year 2013. A theoretical sampling was applied on the basis of the State Midwives Census and the sample size was defined by theoretical saturation of each axis: risk factors, detection and management of high risk pregnancy. The field work began by identifying key informants who then invited midwives to a "State Meeting", working with brainstorming techniques and group interviews. This was followed by 11 indepth interviews with the midwives and a semiotic data analysis.

Results: Overall, 82 informant midwives were included, with a median age of 53 . Of them, $21.95 \%$ were empirical, $42.69 \%$ were traditional trained midwives, and $35.36 \%$ were midwife nurses. In terms of practice, $59.75 \%$ had been practicing for 20 years or more, $63.42 \%$ had primary schooling or less, and $78.05 \%$ had been working for more than 10 years without receiving institutional training. Qualitatively, high risk pregnancy for nurse midwives entails obstetric or neonatal complications, and antenatal control must take place in the hospital. As for traditional empirical and trained midwives, their perspectives include elements of institutional medicine whereby they refer these women to the physician because they assume that they cannot care for those pregnancies. For traditional midwives of indigenous origin, high risk pregnancy represents the "dangerous pregnancy" under the magical-religious model of health.
Conclusions: Midwife perspective regarding high risk pregnancy is determine by traditional medicine and some elements of institutional medicine, differentiated according to the type of midwife and the geographic area where they perform their work. Midwives reported willingness to train in order to contribute to healthy, risk-free pregnancies.

Key words: Midwifery, high risk pregnancy, maternal health, qualitative analysis.

\section{INTRODUCCIÓN}

La reducción de la mortalidad materna es un tema prioritario, por esta razón hace parte de los Objetivos de Desarrollo Sostenible y la Estrategia Mundial para la Salud de la Mujer, el Niño y el Adolescente (1). En consecuencia, México aplica políticas públicas de salud a favor de la maternidad segura, destacando aquellas que buscan alcanzar una oportuna detección y adecuado manejo del embarazo de alto riesgo, al ser esta una condición en la gestación que aumenta la probabilidad de presentar estados patológicos o condiciones anormales concomitantes con la gestación y el parto, que ponen en riesgo la vida de la madre y su hijo (2).

En los países en vías de desarrollo existen distintos factores de riesgo y determinantes sociales que condicionan que las mujeres puedan llevar un embarazo saludable, como la edad y el nivel de educación de la madre, la seguridad social, los riesgos clínicos, la disponibilidad de consultorios o la distancia de la vivienda, la calidad de la atención, su cultura (3) $\mathrm{y}$, sobre todo, aquellos relacionados con vivir en un medio socioeconómico precario (4). Al respecto, organismos internacionales señalan que en estos contextos, con escasos recursos e infraestructura en salud, las parteras adecuadamente entrenadas son esenciales para la detección, derivación y acompañamiento de las mujeres embarazadas, garantizando sus derechos y los del recién nacido $(5,6)$.

En México, la Norma Oficial Mexicana (NOM) 007-SSA2-1993 para la atención de la mujer durante el embarazo, parto y puerperio, y del recién nacido, 
establece que la mayoría de los daños obstétricos pueden ser prevenidos, detectados y tratados con éxito, mediante la aplicación de procedimientos normados para la atención (2). Mediante un control prenatal de alta calidad, Murillo et al. (7) informan una reducción del $80 \%$ de las muertes evitables. Sin embargo, a pesar de ser una prioridad el control prenatal para los países, no todas las mujeres asisten, y quienes lo hacen muestran insatisfacción con el servicio debido a factores personales y barreras de acceso (8), siendo necesario que todo el personal de salud que está en contacto con una mujer embarazada conozca algunos criterios de riesgo que orientan el control prenatal de calidad, incluido el personal no profesional, como son las parteras tradicionales.

Así mismo, el Reglamento de la Ley General de Salud en Materia de Prestación de Servicios de Atención Médica de México, en su artículo 11, señala que el personal no profesional no podrá, en ningún caso, atender los embarazos, partos o puerperios patológicos, excepto cuando la falta de atención en forma inmediata o la transferencia de la paciente a la unidad de atención médica más cercana hagan peligrar la vida de la madre o de su hijo $(9,10)$. De esta manera, y a pesar de esta norma, para el año 2012, la Encuesta Nacional de Salud y Nutrición de México señaló que fueron atendidos 241.000 partos en casa, y uno de cada tres partos fue atendido por parteras empíricas tradicionales (11), sin reconocer que un importante número de ellos eran de alto riesgo, ni las acciones que las parteras deben realizar ante esta situación.

Investigaciones en México documentan que la partería tradicional se mantiene vital aun sin la vinculación con los servicios de salud, principalmente en comunidades rurales, respondiendo a necesidades locales de atención en salud, ejercida en condiciones de extrema pobreza y con poca o nula capacitación institucional (12). Otros estudios señalan que esta práctica tradicional se encuentra subestimada desde el punto de vista social, pues pagar por un médico es un signo de mayor estatus; sin embargo, a pesar de ello aún hay población en
México que acude a los servicios de las parteras tradicionales, tanto en el contexto rural e indígena como en el urbano (6).

En Jalisco, México, la partería es una práctica tradicional vital, con o sin reconocimiento institucional. Para el 2013, de 180 parteras identificadas, el $95 \%$ eran tradicionales y el $89 \%$ se encontraba en la zona rural (13). Por tanto, el sector salud requiere identificar la participación de la partería tradicional en el cuidado del control prenatal de mujeres con embarazo de alto riesgo. Para ello, el presente estudio se planteó el objetivo de comprender la perspectiva de las parteras frente a los factores de riesgo, detección y manejo del embarazo de alto riesgo en Jalisco, México, en el 2013.

\section{MATERIALES Y MÉTODOS}

Diseño. Estudio cualitativo que retoma los principios teóricos metodológicos de la fenomenología, el cual permite explorar cómo representan, aprenden y manejan las parteras el embarazo de alto riesgo, considerando que los significados configurados de manera subjetiva solo se comprenden a partir de la experiencia y perspectiva de las parteras frente a esta condición de salud (14).

Participantes, muestra y muestreo. Para la identificación de las parteras informantes se solicitó al Sector Salud Jalisco el Censo Estatal de Parteras, teniendo un total de 185 registradas al 2010. De este total, se planteó tomar una muestra con la técnica de muestreo teórico (15) que lograra la representatividad de la estructura sociocultural de las trece regiones sanitarias en que se dividen los servicios de salud en Jalisco; se definieron los siguientes perfiles sociodemográficos de las parteras informantes para integrar la muestra: ubicación (urbano-rural), tipo de población (indígena-mestiza) y tipo de parteras (partera enfermera, partera tradicional empírica y partera tradicional capacitada).

En lo que respecta al tamaño de la muestra, se consideró la saturación teórica de la información, es decir, hasta el punto en que ya no se obtiene nueva información o esta comienza a ser redundante (15). 
A partir del objetivo de la investigación se planteó como eje guía de indagación la perspectiva de las parteras frente a los factores de riesgo, detección y manejo del embarazo de alto riesgo, siendo importante para los investigadores la identificación de otras categorías emergentes dentro del trabajo de campo, sobre todo aquello que no se consideró al plantear la investigación.

El trabajo de campo. El estudio se inició en febrero del 2013 y se dio por terminado en agosto del mismo año; considerando que los cuatro investigadores no tenían ninguna relación previa con las parteras, identificaron y se entrevistaron con informantes clave (trece médicos coordinadores de programas de salud en la Secretaría de Salud, cuatro líderes comunitarios locales y una organización de la sociedad civil), quienes una vez conocieron el objetivo del estudio y la forma de trabajo invitaron a las parteras de todo Jalisco a participar de un "Encuentro estatal de parteras", que se llevó a cabo por decisión de informantes clave y algunas parteras e investigadores, en la casa de la cultura de una comunidad rural de Jalisco.

Para la documentación de la información dentro del "Encuentro estatal de parteras", y valorando el perfil de las parteras asistentes, se integraron trece mesas de trabajo, que quedaron conformadas como se describen en la tabla 1, las cuales responden al perfil sociodemográfico establecido por los investigadores (13).

Para guiar el trabajo, un investigador con experiencia en métodos participativos y otro en métodos cualitativos participaron como facilitadores (guía del trabajo metodológico por realizar) y dos investigadores como observadores (toma de registros y apoyo logístico). Dada la naturaleza del objeto de estudio y las características socioculturales de las informantes, se inició el proceso con un trabajo participativo, utilizando la técnica "lluvia de ideas" (16), con la finalidad de poner en común el conjunto de ideas o conocimientos que cada partera tiene sobre el objeto de estudio, utilizando para ello una manta, imágenes, marcadores y recortes

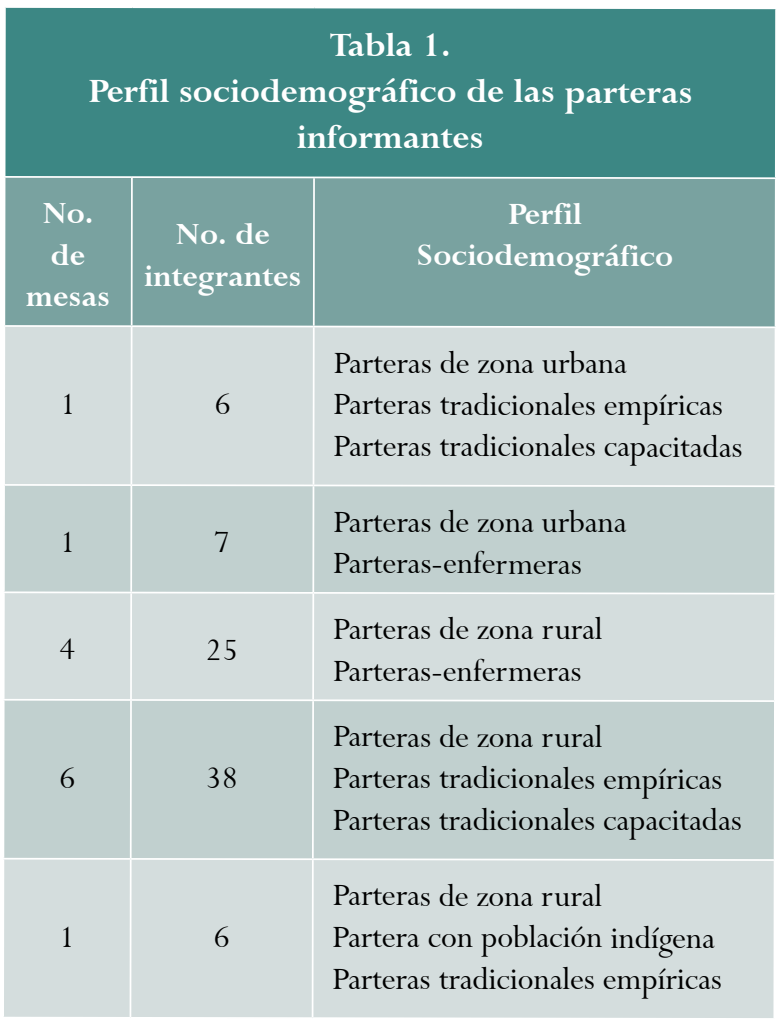

de periódicos, material llevado por las parteras y los investigadores.

Así mismo, con previa autorización de las parteras, se utilizó una grabadora a fin de documentar todo lo que el grupo debatía. Uno de los facilitadores realizó la pregunta ¿qué es embarazo de alto riesgo o de peligro?, permitiendo a las parteras en cada mesa iniciar un proceso de diálogo (técnica de entrevista grupal cara a cara); a su vez, motivados por los mismos facilitadores, fueron colocando en la manta las imágenes, los textos o los dibujos que permitiera plasmar su perspectiva en colectivo. Todo el proceso tuvo una duración de 2 horas, y finalizó con la presentación de los resultados de cada mesa a todos los asistentes.

Con la finalidad de alcanzar la saturación teórica de la información, el grupo de investigadores seleccionó de las mesas de trabajo a once parteras, cinco parteras tradicionales empíricas de zona rural y seis parteras tradicionales capacitadas, también de zona rural, a quienes dos investigadores visitaron en sus domicilios para realizar una entrevista individual a 
profundidad, indagando el mismo eje temático; las entrevistas se realizaron durante tres o cuatro visitas de una hora cada una, utilizando la grabadora para captar la información, así como el diario de campo al término de cada día, para registrar notas teóricas, metodológicas y analíticas, lo cual permitió discutir y determinar la saturación de los datos entre los investigadores.

Confiabilidad y análisis de los datos. Para lograr la confiabilidad de los datos se consideraron diversos aspectos citados por algunos autores: a) mantener una observación persistente y prolongada durante todo el trabajo, b) se citaron otros estudios realizados y la normatividad en el tema al inicio y al final del trabajo, c) se devolvieron los resultados a un grupo de parteras informantes, convocadas en una comunidad rural por informantes clave para corroboración y afirmación de los hallazgos plasmados, y d) comprobación de saturación de datos por los miembros de la investigación (17).

Las grabaciones de las entrevistas grupales e individuales fueron transcritas a textos de Word y, junto con la imágenes, los textos y dibujos generados dentro de las mesas, se analizaron e interpretaron bajo el modelo actancial semiótico, lo que implicó la realización de lectura y relectura de cada línea textual, para pasar a describir primeras impresiones contextualizadas tanto de ejes temáticos ya considerados, como de temas emergentes, pasando al análisis de la superficie de los textos, con identificación de actores semióticos, isotopías temáticas y figurativas, así como la dimensión cognitiva, para generar una estructuración del texto en grandes categorías con descripciones textuales que documentaran la perspectiva de las parteras $(18,19)$.

La participación de las parteras fue voluntaria y bajo consentimiento informado, con respeto por la autonomía, autodeterminación y confidencialidad de la información, con el protocolo dictaminado por el Comité de Investigación y el Comité de Ética en Investigación de la Oficina Central de Secretaría de Salud Jalisco. Se cumplieron los criterios metodo- lógicos, éticos y de originalidad, de acuerdo con la Norma Técnica 313, con registro 57/RIV-JAL/2012.

\section{RESULTADOS}

En el estudio participaron un total de 82 parteras, de 42 municipios de las 13 regiones sanitarias en las que divide a Jalisco el Sector Salud; en cuanto a las características sociodemográficas de las parteras informantes, la mediana de edad fue de 53 años (rango de 17 a 72 años), siendo 18 parteras tradicionales empíricas (21,95\%), un total de 35

\begin{tabular}{|c|c|c|}
\hline \multicolumn{3}{|c|}{$\begin{array}{c}\text { Tabla } 2 . \\
\text { Características sociodemográficas de parteras } \\
\text { en Jalisco, México, } 2013\end{array}$} \\
\hline Caracteristícas & f & $\%$ \\
\hline \multicolumn{3}{|c|}{ Años de ser partera } \\
\hline$<5$ años & 3 & 3,65 \\
\hline 5 a 9 años & 6 & 7,34 \\
\hline 10 a 14 años & 10 & 12,19 \\
\hline 15 a 19 años & 14 & 17,07 \\
\hline 20 años o más & 49 & 59,75 \\
\hline \multicolumn{3}{|c|}{ Nivel de estudios } \\
\hline Analfabeta & 22 & 26,83 \\
\hline Primaria & 30 & 36,59 \\
\hline Secundaria & 12 & 14,63 \\
\hline Bachillerato & 6 & 7,31 \\
\hline Carrera técnica & 4 & 4,88 \\
\hline Licenciatura & 8 & 9,76 \\
\hline \multicolumn{3}{|c|}{$\begin{array}{l}\text { Años transcurridos desde la última } \\
\text { capacitación por el sector salud }\end{array}$} \\
\hline Más de 10 años & 64 & 78,05 \\
\hline De 5 a 9 años & 12 & 14,64 \\
\hline Menos de 5 años & 6 & 7,31 \\
\hline Total & 82 & 100 \\
\hline
\end{tabular}

Fuente: investigación de campo. 
parteras tradicionales capacitadas (42,69\%) y 29 parteras enfermeras $(35,36 \%)$; los años de ejercicio como parteras, el nivel de estudios y el tiempo transcurrido desde su última capacitación por el sector salud se presentan en la tabla 2.

La perspectiva de las parteras en Jalisco frente al embarazo de alto riesgo se configura a partir del tipo de partera, así como al contexto geográfico y cultural donde se desempeña; a continuación se describen los resultados de estos supuestos.

La partera-enfermera y el embarazo de alto riesgo. La característica de estas mujeres es que siendo parteras tradicionales, estudiaron la carrera de enfermería, o algunas, siendo enfermeras y al entrar en contacto con parteras tradicionales, adoptaron la práctica de la partería; sin embargo, para el sector salud todas ellas son solo enfermeras, mientras que para la comunidad son enfermeras y también parteras, lo cual dificulta calificarlas en el presente estudio como parteras profesionales. Para este tipo de parteras, el embarazo de alto riesgo representa una condición clínica donde se presentan complicaciones obstétricas y neonatales que pueden llevar a una muerte materna o neonatal.

Las parteras-enfermeras consideran que el control del embarazo, parto y puerperio debe hacerse solo en unidades hospitalarias, pero señalan que algunas de ellas hacen visita a los hogares de las pacientes para conocer si acuden a sus controles prenatales programados, o cuando la paciente acude a ellas por presentar alguna alteración o malestar asociado al embarazo; algunos de estos constructos se representan en el siguiente discurso retomado de las entrevistas: "solo podemos atender embarazos y partos normales, no los de riesgo, eso lo hacen en el hospital con médicos preparados" (partera-enfermera).

$\mathrm{Al}$ explorar los factores que llevan a una mujer embarazada a presentar un embarazo de alto riesgo, se identifica en sus discursos la incorporación tanto de elementos de la medicina institucional como de

\begin{tabular}{|c|c|c|}
\hline Categoría & $\begin{array}{c}\text { Elementos desde la cosmovisión de la } \\
\text { medicina tradicional }\end{array}$ & $\begin{array}{l}\text { Elementos desde la cosmovisión } \\
\text { de la medicina institucional }\end{array}$ \\
\hline $\begin{array}{l}\text { Factores de riesgo } \\
\text { para presentar un } \\
\text { embarazo de alto } \\
\text { riesgo o "complicado" }\end{array}$ & $\begin{array}{l}\text { - "Eclipse" } \\
\text { _ "Antojos no cumplidos" } \\
\text { _ "Mal posición del niño" }\end{array}$ & $\begin{array}{l}\text { - "Tener más de } 45 \text { años (añosa)" } \\
\text { _ "Tener menos de } 15 \text { años (primeriza)" } \\
\text { _ "Presión arterial alta" } \\
\text { _ "Diabética" } \\
\text { - "Abortos" } \\
\text { - "Muchos hijos" } \\
\text { - "Cesáreas" }\end{array}$ \\
\hline $\begin{array}{l}\text { Detección del } \\
\text { embarazo de alto } \\
\text { riesgo o "complicado" }\end{array}$ & $\begin{array}{l}\text { - "Disminución del líquido por eclipses" } \\
\text { - "Dolor del vientre o que se mueva } \\
\text { mucho si no cumple antojo" } \\
\text { - "Dolor en las noches de que se encaja } \\
\text { el niño por enojo" }\end{array}$ & $\begin{array}{l}\text { - "Hinchazón de los pies" } \\
\text { - "Dolor fuerte de cabeza" } \\
\text { - "Se le taponean o zumban los oídos" } \\
\text { - "Traer el azúcar alta" } \\
\text { - "Suben mucho de peso" } \\
\text { - "Desecho vaginal cafesoso y oloroso" }\end{array}$ \\
\hline $\begin{array}{l}\text { Manejo del embarazo } \\
\text { de alto riesgo o } \\
\text { "complicado" }\end{array}$ & $\begin{array}{l}\text { - "Ponerse un listón o algo rojo en el } \\
\text { eclipse" } \\
\text { _ "Traer diario un alfiler o algo de fierro" } \\
\text { _ "Que cumpla sus antojos" } \\
\text { _ "La sobada, mínimo cada mes" }\end{array}$ & $\begin{array}{l}\text { _ "Tener chequeo con el médico especialista" } \\
\text { _ "Toma de laboratoriales" } \\
\text { _ "Pesarla, tomar el azúcar y la presión cada } \\
\text { mes" }\end{array}$ \\
\hline
\end{tabular}

Fuente: tomado de las entrevistas. 
otros que corresponden a la cosmovisión sociocultural de la medicina tradicional (tabla 3), encontrando que, para algunas parteras-enfermeras, estos factores tienen la misma importancia al momento de emitir una valoración del estado de salud de la mujer embarazada y sus posibles complicaciones.

En algunas parteras informantes de este grupo, los saberes que responden a la medicina institucional fueron adquiridos dentro de su formación como enfermeras, pero continúan retroalimentándose durante las capacitaciones que reciben por el sector salud, enfatizando que participan por ser enfermeras y no como parteras. Las enfermeras que aprendieron a atender partos con otra partera tradicional han incorporado estos saberes populares de la salud materna y perinatal dentro del ejercicio de acompañamiento que le brindan las parteras tradicionales en la atención de mujeres embarazadas dentro de la localidad donde viven, población para la cual, además de ser enfermeras, también son parteras.

Finalmente, expresan que en su experiencia han tenido situaciones donde la mujer con un diagnóstico médico establecido de embarazo de alto riesgo son irregulares en sus citas al hospital o simplemente no acuden, aludiendo que esto se debe a no tener dinero para el traslado, que la comunidad está muy alejada del hospital, que a la pareja no le gusta que la mujer sea vista o tocada por otro hombre y, en otros casos, la pareja no le permite acudir a ninguna unidad de salud institucional por presentar marcas de golpes en el cuerpo, situaciones ante las cuales la partera-enfermera se siente obligada a brindarles atención dentro de sus posibilidades, algo que se ejemplifica en el siguiente texto tomado de las entrevistas: "Tenemos mujeres con embarazo de alto riesgo que nunca van al hospital o pocas veces... no tienen para comer, menos para ir cada mes a Guadalajara, por eso las sigo atendiendo yo, no puedo dejarlas sin revisión; iay!, le pido ayuda a Dios y la virgen para que todo salga bien" (partera-enfermera).

La partera tradicional-capacitada frente al embarazo de alto riesgo. Estas parteras se han iniciado en la práctica tradicional de la partería, principalmente desde la adolescencia, pero durante el transcurso de los años han asistido a capacitaciones que imparten el sector salud y organizaciones de la sociedad civil. Los servicios de atención a la embarazada antes, durante y después del parto los prestan en su domicilio particular, y solo algunas entregan información de sus actividades al sector salud.

\begin{tabular}{|c|c|c|}
\hline Categoría & $\begin{array}{l}\text { Elementos desde la cosmovisión } \\
\text { de la medicina tradicional }\end{array}$ & $\begin{array}{l}\text { Elementos desde el conocimiento } \\
\text { de la medicina institucional }\end{array}$ \\
\hline $\begin{array}{l}\text { Factores de riesgo para } \\
\text { presentar un embarazo de } \\
\text { alto riesgo o "de peligro" }\end{array}$ & $\begin{array}{l}\text { - "Eclipse” } \\
\text { - "Mal acomodo del niño" } \\
\text { - "Marido machista" }\end{array}$ & $\begin{array}{l}\text { - "Mujer añosa” } \\
\text { - "Que se le suba la presión” } \\
\text { _ "Que tenga azúcar“" }\end{array}$ \\
\hline $\begin{array}{l}\text { Detección del embarazo de } \\
\text { alto riesgo o "de peligro" }\end{array}$ & $\begin{array}{l}\text { - "Le lastima al dormirse o acostar- } \\
\text { se el niño cuando se encaja" }\end{array}$ & $\begin{array}{l}\text { - "Pies como de sapo" } \\
\text { - "Primer parto" } \\
\text { - "Le chillan los sentidos" } \\
\text { - "Se sube su presión" }\end{array}$ \\
\hline $\begin{array}{l}\text { Manejo del embarazo } \\
\text { de alto riesgo }\end{array}$ & $\begin{array}{l}\text { - "El listón rojo y su alfiler" } \\
\text { - "La sobada de cada mes" }\end{array}$ & $\begin{array}{l}\text { - "Que vaya con el doctor" } \\
\text { _ "Pedirle que se tome su presión y su azúcar" }\end{array}$ \\
\hline
\end{tabular}

Fuente: tomado de las entrevistas. 
En estas parteras se identifican elementos de la medicina institucional y tradicional, descritos en la tabla 4; no obstante, señalan que a pesar de representar el embarazo de alto riesgo como un "embarazo de peligro" o "embarazo de cuidado", en su práctica adquiere mayor sentido los que responden a la medicina tradicional, utilizando los aspectos conceptuales y prácticos de la medicina institucional solo para detectar y enviar al médico a las mujeres embarazadas que ellas sospechen que tenga un embarazo de alto riesgo, dado que ellas no pueden atender ese tipo de embarazos.

Describen que cuando tienen que atender a mujeres embarazadas con diagnóstico establecido de embarazo de alto riesgo, algunas parteras les piden su "tarjeta de atención del médico", pues es muy importante para ellas conocer si la paciente está siendo atendida en una unidad de salud institucional, refiriendo que en su experiencia, algunas mujeres no acuden a su seguimiento con el médico.

Ante lo anterior, las parteras consideran importante que el sector salud las forme para atender a estas mujeres, y manifiestan su disposición de formarse y poder colaborar con el sector a fin de lograr embarazos saludables y sin riesgos, enfatizando sobre todo en la posibilidad de que sean reconocidas como parteras por el personal de salud al llevar a una embarazada a una unidad hospitalaria, ya que en su experiencia han sido rechazadas, situación ejemplificada en el siguiente texto de entrevista: "Pues no más nos dicen que no atendamos mujeres con embarazo de peligro, pero muchas nos llegan con eso y ni modo que no la atendamos, además de que luego las lleva uno al hospital y me rechazan por ser partera" (partera-tradicional capacitada).

La partera tradicional-empírica frente al embarazo de alto riesgo. Este tipo de parteras son mujeres rurales, sin estudio o con estudios de primaria, que nunca han recibido capacitación del sector salud o alguna otra instancia, quienes ejercen en su mayoría de manera oculta y en sus domicilios particulares. Estas parteras también entienden el embarazo de alto riesgo como un "embarazo de peligro", pero a diferencia de las parteras tradicionales capacitadas, este peligro está vinculado a aspectos mágicoreligioso, sin identificar elementos de la medicina institucional, solamente algunos que tienen que ver con condiciones de la mujer: la edad y el estado nutricional, así como el edema de extremidades, este último no vinculado a causas fisiológicas sino a otras causas de índole tradicional. Estos datos se describen de manera textual en la tabla 5 .

Parteras tradicionales de comunidades con población indígena. Al inicio de las entrevistas con este tipo de parteras no se identificaron elementos que evidenciaran conocimientos relacionados con el embarazo de alto riesgo, visto desde el modelo médico institucional; no obstante, al retomar algunos conceptos propios de estas parteras, se replanteó la pregunta generadora: ¿qué es embarazo de peligro y qué cuidados se deben tener en una mujer durante su embarazo? A partir de esta pregunta se describe una serie de condiciones durante el embarazo que se configuran en el modelo mágico-religioso de la salud, aspectos que están impregnados de la identidad cultural del grupo social local donde ejercen su práctica como parteras.

Es importante señalar que a pesar de ser concepciones tradicionales de la salud, dentro de su actuar se identifican algunos elementos que responden a la visión de la medicina institucional, los cuales se encuentran vinculados y arraigados a los aspectos tradicionales, tal como se muestra en la tabla 6.

\section{DISCUSIÓN}

De las características de las parteras informantes se destaca su baja escolaridad y ser mujeres de edad avanzada. Si se tienen en cuenta las Normas Internacionales (6) para considerar la posibilidad de su profesionalización (haber completado educación secundaria y estudiar por tres años la profesión), se hacen evidentes las dificultades para que obtengan su licencia como parteras profesionales cualificadas y así poder participar en la detección y el manejo de mujeres con embarazo de alto riesgo. Esta situación 
Tabla 5.

Saberes y perspectiva de parteras tradicionales-empíricas frente al embarazo de alto riesgo en Jalisco, México, 2013

\begin{tabular}{|c|c|c|}
\hline Categoría & $\begin{array}{l}\text { Elementos desde la cosmovisión } \\
\text { de la medicina tradicional }\end{array}$ & $\begin{array}{c}\text { Elementos desde el } \\
\text { conocimiento de la medicina } \\
\text { institucional }\end{array}$ \\
\hline $\begin{array}{l}\text { Factores de riesgo para } \\
\text { presentar un embarazo de } \\
\text { alto riesgo o "de peligro" }\end{array}$ & $\begin{array}{l}\text { - "Antojos" } \\
\text { - "Envidias y corajes" } \\
\text { _ "Eclipse" } \\
\text { - "Asistir a velorios o entierros" } \\
\text { - "Maleficio a ella o el niño" }\end{array}$ & $\begin{array}{l}\text { - "Estar tiernitas y que sea su } \\
\text { primer embarazo" } \\
\text { _ "Que estén desnutridas" }\end{array}$ \\
\hline $\begin{array}{l}\text { Detección del embarazo de } \\
\text { alto riesgo o "de peligro" }\end{array}$ & $\begin{array}{l}\text { - " Dolor de estómago por los antojos o corajes" } \\
\text { _ "No se puede dormir y sueña cosas de muerte" } \\
\text { _ "Se le sale el agua" } \\
\text { _ "Le pasan cosas feas o malas en la casa" } \\
\text { - "Que no se le mueva" }\end{array}$ & $\begin{array}{l}\text { - "Pies como de sapo por la } \\
\text { bilis de corajes o no cumplir } \\
\text { antojos" }\end{array}$ \\
\hline $\begin{array}{l}\text { Manejo del embarazo de alto } \\
\text { riesgo }\end{array}$ & $\begin{array}{l}\text { _ "El listón rojo y su alfiler" } \\
\text { _ "Que cumpla sus antojos, y si no tiene dinero } \\
\text { que coma tierra o lodo" } \\
\text { - "Acomodo del niño al mes o cuando lo sienta } \\
\text { atravesado" } \\
\text { _ "Que no acuda a velorios o entierros" }\end{array}$ & \\
\hline
\end{tabular}

Fuente: tomado de las entrevistas.

Tabla 6.

Saberes y perspectiva de parteras frente a un embarazo "de peligro" en comunidades con población indígena de Jalisco, México, 2013

\section{Textos representativos}

- "Sangrado de que se viene el niño"

- "Coso tres cogollitos de chile mirasol y algo de oro, que se lo tome, que se coma su caldo de pollo con mucho jitomate y chayote pa que agarre fuerzas"

- "Tener la matriz inmadura"

- "Se le inyecta cuerpo amarillo, 1 cada 3 días hasta que complete 5 que son las que trae la cajita"

- "Le hacen el mal a ella y su niño"

- "Uno se da cuenta porque le canta el tecolote, para eso se le hace una limpia y sacarle lo malo, para eso aquí hay quien lo haga"

Fuente: tomado de las entrevistas. es diferente para las parteras-enfermeras, a quienes a pesar de tener perfil de parteras profesionales, el sector salud solo las considera como enfermeras.

Se destaca que el 78,05\% de las informantes tienen más de 10 años sin haber recibido alguna capacitación por el sector salud, lo cual se explica porque en gran medida su perspectiva frente al embarazo de alto riesgo está inscrita en el modelo mágico-religioso de la salud, situación encontrada también en parteras de San Luis Potosí, México, quienes mencionan como factores de riesgo la “caída de la matriz", "el bebé parado" (posición podálica) y el "nacimiento de gemelos" (20), con el riesgo de que la partera no capacitada no perciba la importancia de los antecedentes ginecoobstétricos patológicos o signos de alarma, situación documentada en parteras rurales de Morelos, México (21).

En las parteras que viven con población indígena, su perspectiva frente al embarazo de alto riesgo no 
se fundamenta en principios de la medicina institucional, sino que se configura dentro de la cosmovisión tradicional del proceso salud-enfermedad propio del contexto sociocultural donde ejercen su práctica, reconociendo y valorando la diversidad de elementos socioculturales determinantes de la salud en las mujeres indígenas: condición de género, nivel de estudios, posición económica, redes comunitarias y los significados culturales de ser mujer y ser madre en una población indígena, elementos que las parteras reconocen e incorporan en su práctica (22).

Respecto a la participación de la partera tradicional en comunidades con población indígena, algunas investigaciones señalan que la cobertura del Seguro Popular en México hizo que la atención hospitalaria del parto en mujeres indígenas aumentara del 63,8 a 76,4\%, a diferencia de la población no indígena donde se tienen cifras de 93,9\% de atención hospitalaria (23), por lo que el sector salud debe tener un mayor acercamiento a estas parteras, sobre todo para poder establecer espacios de enriquecimiento mutuo de saberes y prácticas frente a la atención del embarazo, parto y puerperio, con énfasis en lo relacionado con el embarazo de alto riesgo.

Las parteras tradicionales que estudiaron la carrera de enfermería y se convirtieron en trabajadoras institucionales de salud tienen la oportunidad de obrar en conjunto con otros profesionales del sector, pero también, pueden ser parte de las capacitaciones del sector salud en temáticas relacionadas con la salud materna y perinatal, incluido el embarazo de alto riesgo, lo cual puede influir para modificar su perspectiva tradicional. Esta situación también se documentó en población de Colombia (24), donde las parteras tradicionales realizaron cursos de enfermería en la Cruz Roja Colombiana y otras organizaciones no gubernamentales, cambiando su actuar frente a la mujer embarazada y al embarazo de alto riesgo.

En cuanto a las parteras tradicionales-capacitadas y empíricas, el hecho de que su práctica no sea reconocida por el sector salud, las limita para recibir las capacitaciones que otorga a los profesionales de la salud referentes al embarazo de alto riesgo, lo cual refuerza que en su perspectiva se identifiquen más elementos de la medicina tradicional que de la occidental. Frente a este distanciamiento, descrito ya por otras investigaciones $(25,26)$, es necesario generar encuentros de inclusión que permitan la sensibilización y posibilidad de tener un trabajo conjunto, ratificando las funciones esenciales que cada uno de los participantes realiza o puede realizar en torno al embarazo de alto riesgo.

Aunado a lo anterior, debe valorarse que a pesar de la resistencia de las parteras a la crítica médica, el proceso de la medicalización del embarazo y el parto en México ha hecho que las parteras tradicionales incorporen en su perspectiva frente al embarazo de alto riesgo algunos elementos de la medicina institucional, conservando aún gran parte de su saber tradicional, saberes y prácticas que asumen como esenciales frente al proceso salud-enfermedad y la salud materna, en el contexto pluricultural del país, por lo que gran parte del éxito del trabajo de capacitación que otorgue el sector salud está en valorar y no invalidar esta cosmovisión (25).

A pesar de existir reglamentación para la profesionalización de la práctica de la partería en México, la vitalidad de la partera tradicional y empírica es un aspecto que deberá ser analizado por el sector salud, tomando en cuenta la experiencia de otros contextos como África y Asia, donde se han identificado barreras económicas que limitan el acceso a la atención institucional del embarazo y parto en contexto rural, contraponiéndose con la cercanía y confianza que tienen las mujeres con parteras tradicionales, quienes han vivido toda su vida en la comunidad, hablan el dialecto local y comparten elementos socioculturales de la salud materna y perinatal (27).

En el caso de los países europeos se destaca un trabajo bajo estrictas normas de seguridad y vigilancia de la práctica de la partería por parte del 
sector salud, un entrenamiento especializado, con el reconocimiento de la comunidad médica del papel de la partera en la reducción de la mortalidad materna $(28,29)$, acciones que en México aún no están claras, sobre todo con las parteras no profesionales, lo cual dificulta en gran medida dar cumplimiento a los derechos sexuales y reproductivos, al no darles reconocimiento y formación constante que influya en su perspectiva y actuar frente al embarazo de alto riesgo (30).

Además de lo anterior, deben valorarse experiencias propias de México por mejorar la participación de la partería frente al embarazo de alto riesgo, por ejemplo en San Luis Potosí, donde se creó un programa integral para reducir la mortalidad materna en el quinquenio 2004-2008 (31), que incluyó un modelo de partería profesional en un hospital comunitario y dos hospitales generales, con la intención de humanizar el parto e incrementar la calidad de la atención, logrando que el modelo fuera aceptado por las parteras, la población y el sector salud; quedó pendiente la asignación constante y progresiva de los recursos públicos para estas acciones.

Las acciones que busquen modificar la perspectiva de la partería frente al embarazo de alto riesgo deben destacar el trabajo en conjunto y la formación constante, donde las parteras realmente se apropien de los conocimientos y puedan determinar hasta dónde y cómo actuar frente a una mujer con embarazo de alto riesgo, evitando el predominio de un modelo de atención sobre el otro pues, de lo contario, las parteras que participen solo lo harán para obtener el "carné" y evitar ser penalizadas por practicar de forma ilegal, pues perciben que con ello aumentan la legitimidad de su trabajo por su proximidad con los valores biomédicos, pero no modifican su perspectiva y actuar, poniendo en riesgo la vida de la madre y el recién nacido (32).

Es importante destacar que los datos que aquí se presentan muestran solo la perspectiva de las parteras que decidieron voluntariamente participar del estudio, teniendo pendiente aún conocer el punto de vista de las parteras que deciden no tener contacto con el sector salud. Así mismo, estos hallazgos pueden servir de base para llevar a cabo investigaciones desde un enfoque cuantitativo, con el diseño de instrumentos que permitan medir el nivel de conocimientos de las parteras frente al embarazo de alto riesgo, tomando siempre en cuenta la naturaleza del objeto de estudio.

\section{CONCLUSIONES}

La perspectiva de las parteras frente al embarazo de alto riesgo está determinada principalmente por la medicina tradicional, pero con algunos elementos de la medicina institucional, diferenciada según el tipo de partera y la zona geográfica en que ejerce su práctica. En la configuración de esta perspectiva influye también el trabajo en conjunto con personal de salud, pues las parteras están dispuestas a recibir formación que mejore su actuar y la toma de decisiones ante el embarazo de alto riesgo. Así mismo, es necesario establecer mecanismos para que las parteras puedan remitir a estas embarazadas a un personal cualificado para su manejo y así evitar que terminen en una muerte materna o neonatal.

\section{REFERENCIAS}

1. Organización Mundial de la Salud. Mortalidad materna. Centro de Prensa. Nota descriptiva. Septiembre de 2016 [visitado 2017 Feb 02]. Disponible en: http:// www.who.int/mediacentre/factsheets/fs348/es/

2. Estados Unidos Mexicanos, Secretaría de Salud, Comité Consultivo Nacional de Normalización de Servicios de Salud. Norma Oficial Mexicana (NOM007-SSA2-1993), Atención de la mujer durante el embarazo, parto y puerperio y del recién nacido. Criterios y procedimientos para la prestación del servicio [visitado 2017 Feb 02]. Disponible en: http:// www.salud.gob.mx/unidades/cdi/nom/007ssa23.html

3. Say L, Raine R. A systematic review of inequalities in the use of maternal health care in developing countries: examining the scale of the problem and the importance of context. Bull World Health Organ. 2007;85:812-9. 
4. Cáceres FM, Molina G. Inequidades Sociales en Atención Materna. Rev Colomb Obstet Ginecol. 2010 [visitado 2017 Feb 02];61(3). Disponible en: http:// www.scielo.org.co/pdf/rcog/v61n3/v61n3a07.pdf

5. Alarcon MA, Sepulveda J, Alarcon IC. Las parteras, patrimonio de la humanidad. Rev Colomb Obstet Ginecol. 2011 [visitado 2017 Feb 02];62(2). Disponible en: http://www.scielo.org.co/pdf/rcog/ v62n2/v62n2a10.pdf

6. Fondo de Población de las Naciones Unidas (UNFPA). El estado de las parteras en el mundo 2014. Hacia el acceso universal a la salud, un derecho de la mujer. 2014 [visitado 2017 Feb 02]. Disponible en: http://www.unfpa.org/sites/default/files/pub-pdf/ SoWMy2014_complete-Spanish.pdf

7. Murillo L, Miranda W. Calidad del control prenatal en el Centro de Salud Ciudad Sandino, Managua. Agostooctubre 2004 (Tesis). Managua: Universidad Nacional Autónoma de Nicaragua. Centro de Investigaciones y Estudios de la Salud; 2004 [visitado 2017 Feb 02]. Disponible en: http://cedoc.cies.edu.ni/digitaliza/ t289/t289.htm

8. Cáceres FM. El control prenatal: una reflexión urgente. Rev Colomb Obstet Ginecol. 2009 [visitado 2017 Ene 15];60(2). Disponible en: http://revista. fecolsog.org/index.php/rcog/article/viewFile/342/358

9. Secretaría de Salud. Reglamento de la Ley General de Salud en materia de prestación de Servicios de Atención Médica. Reglamento publicado en el DOF el 14 de mayo de 1986. Texto Vigente última reforma publicada DOF 19-12-2016 [visitado 2017 Feb 01]. Disponible en: http://www.cgajdh.salud.gob.mx/ descargas/LV/18-RLGSSAM-DOF_19-12-16.pdf

10. Secretaría de Salud. Gobierno Federal. Guía para la autorización de las parteras tradicionales como personal de salud no profesional [visitado 2017 Ene 20]. Disponible en: http://www.dgplades.salud.gob. $\mathrm{mx} /$ Contenidos/Documentos/MedicinaTradicional/ GuiaAutorizacionParteras.pdf

11. Lazcano E, Schiavon R, Uribe P, Walker D, Suárez L, Luna R, Ulloa A. Cobertura de atención del parto en México: su interpretación en el contexto de la mortalidad materna. Salud Pública de México. 2013 [visitado 2017 Feb 02];55. Disponible en: http://www. scielo.org.mx/pdf/spm/v55s2/v55s2a19.pdf

12. Laureano J, Mejía ML, Villaseñor M, Gil E. Experiencia de trabajo con parteras en Jalisco. Salud pública de México. 2014 [visitado 2017 Feb 02]; 56(6). Disponible en: http://www.scielo.org.mx/pdf/spm/ v56n6/v56n6a1.pdf

13. Laureano J, Villaseñor M, Mejía ML, Ramírez H. Ejercicio tradicional de la partería frente a su profesionalización: estudio de caso en Jalisco, México. Facultad Nacional de Salud Pública. 2016 [visitado 2017 Feb 02];34(3). Disponible en: http://aprendeenlinea.udea.edu.co/revistas/index.php/fnsp/article/ view/21179/20782734

14. Mercado FJ, Gastaldo D, Calderón C. Paradigmas y diseños de la investigación cualitativa en salud. Una antología Iberoamericana. México: Universidad de Guadalajara, Universidad Autónoma de Nuevo León, Servicio Vasco de Salud Osakidetza y Asociación Médica de Jalisco; 2002. p. 439-59.

15. Martín C, Salamanca AC. El muestreo en la investigación cualitativa. Nure Investigación. 2007 [visitado 2017 Feb 02];27. Disponible en: http://www.sc.ehu. es/plwlumuj/ebalECTS/praktikak/muestreo.pdf

16. Bustillos G, Vargas L. Técnicas participativas para la educación popular. 4 ed. Guadalajara: IMDEC; 1998.

17. Santaella CM. Criterios de validez en la investigación cualitativa actual. Revista de Investigación Educativa. 2006 [visitado 2017 Feb 02];24(1). Disponible en: http://revistas.um.es/rie/article/viewFile/97351/93461

18. Karam T. Introducción a la semiótica. Portal de la Comunicación InCom-UAB: el portal de los estudios de comunicación, 2001-2011. [visitado 2017 Feb 02]. Disponible en: http://www.portalcomunicacion.com/ uploads/pdf/18_esp.pdf

19. Greimas AJ, Campodónico H, Courtés J, Ballón E. Semiótica: diccionario razonado de la teoría del lenguaje. Madrid: Gredos; 1990 [visitado 2017 Feb 02]. Disponible en: http://dialnet.unirioja.es/servlet/ libro? codigo $=380477$

20. Pelcastre B, Villegas N, De León V, Díaz A, Ortega D, Santillana M, et al. Embarazo, parto y puerperio: 
creencias y prácticas de parteras en San Luis Potosí, México. Rev Esc Enferm USP. 2005 [visitado 2017 Feb 02];39(4). Disponible en: http://www.scielo.br/ pdf/reeusp/v39n4/01.pdf

21. García C, Castañeda X, Romero X, González D, Langer A. Percepción de las parteras sobre factores de riesgo reproductivo. Salud Pública de México. 1993 [visitado 2017 Feb 02];35(1). Disponible en: http://bvs.insp.mx/rsp/_files/File/1993/ene_feb/ ene_fen_93_08percepcion.pdf

22. Mejía ML, Ortiz RC, Laureano J, Alcántara ECG, López MC, Gil E. Aspectos sociales de la muerte materna: análisis de 5 años en el Hospital General de Occidente: Jalisco, México. Revista chilena de obstetricia y ginecología . 2013 [visitado 2017 Feb 02];78(6). Disponible en: http://www.scielo.cl/pdf/rchog/v78n6/art03.pdf

23. Leyva R, Infante C, Gutiérrez JP, Quintino F. Inequidad persistente en salud y acceso a los servicios para los pueblos indígenas de México, 2006-2012. Salud Pública de México. 2013 [visitado 2017 Feb 02];55. Disponible en: http://www.scielo.org.mx/pdf/spm/ v55s2/v55s2a8.pdf

24. Laza C, Ruiz CH. Entre la necesidad y la fe: la partera tradicional en el Valle del río Cimitarra. Ciencia y enfermería. 2010 [visitado 2017 Feb 02];16(1). Disponible en: http://www.scielo.cl/pdf/cienf/v16n1/ art_08.pdf

25. Jiménez S, Pelcastre B, Figueroa JG. Parteras tradicionales y su relación con las instituciones de salud. Entre la resistencia y la subordinación. Revista Chilena de Salud Pública. 2008 [visitado 2017 Feb 02];12(3). Disponible en: http://www. revistasaludpublica.uchile.cl/index.php/RCSP/article/ viewFile/2205/208526. Comité Promotor por una Maternidad Segura en México. El estado de las parteras en el mundo 2014: oportunidades y retos para México. Ciudad de México: Medios Comunes; 2014 [visitado 2017 Feb 02]. Disponible en: http://www.unfpa.org. $\mathrm{mx} /$ publicaciones/Las_parteras_del_mundo.pdf

27. Laza C. Factores relacionados con la preferencia de las mujeres de zonas rurales por la partera tradicional. Revista Cubana de Salud Pública. 2015 [visitado 2017 Feb 02];41(3). Disponible en: http:// scielo.sld.cu/pdf/rcsp/v41n3/spu08315.pdf

28. Likis FE, King TL, Murphy PA. Perspectives on midwifery regulation. Journal of midwifery \& women's health. 2010 [visitado 2017 Feb 02];55. Disponible en: http://onlinelibrary.wiley.com/doi/10.1016/j. jmwh.2010.07.007/abstract

29. Ward L, Fenton K, Maher L. The high impact actions for nursing and midwifery. 3: Staying safe, preventing falls. Nurs Times. 2010 [visitado 2017 Feb 02];106. Disponible en: http://europepmc.org/abstract/ med/20718370

30. Organización Mundial de la Salud, Organización Panamericana de la Salud. De vuelta a los orígenes del nacimiento. Perspectivas de Salud. 2002 [visitado 2017 Feb 02];6(2). Disponible en: http://www.paho. org/spanish/dpi/Numero12_article2_1.htm

31. Robledo FJ. Reducción de la mortalidad materna en San Luis Potosí. Ginecol Obstet Mex. 2011 [visitado 2017 Feb 02];79(6). Disponible en: http:// www.medigraphic.com/pdfs/ginobsmex/gom-2011/ gom116e.pdf

32. Fleischer S. Pasando por comadrona, midwife y médico: el itinerario terapéutico de una embarazada en Guatemala. Anthropologica. 2006 [visitado 2017 Feb 02];24(24). Disponible en: http://www.scielo.org. pe/pdf/anthro/v24n24/a03v24n24.pdf 